Published by Al-Nahrain College of Medicine P-ISSN 1681-6579

E-ISSN 2224-4719

Email: iraqijms@colmed-alnahrain.edu.iq

http://www.colmed-alnahrain.edu.iq

http://www.iraqijms.net

Iraqi JMS 2019; Vol. 17(1)

\title{
Correlation Between Cardiac and Hepatic T2* MRI and Serum Ferritin Level in Patients with Transfusion dependent $\beta$-Thalassemia Major
}

\author{
Ahmed K. Yaseen ${ }^{1} M B C h B$, Sawsan S. Abbas ${ }^{2} C A B P$, Associate MRCPCh, Ahmed M.A. Abdulhadi ${ }^{1}$ \\ FICP
}

${ }^{1}$ Dept. of Pediatrics, Al-Imamein Al-Kadhimein Medical City, Baghdad, Iraq, ${ }^{2}$ Dept. of Pediatrics, College of Medicine, Al-Nahrain University, Baghdad, Iraq

\begin{abstract}
Background $\quad \beta$-Thalassemia occurs in higher frequency in the Mediterranean area and the Middle East. Classically serum ferritin and liver biopsy have been needed to monitor patient's response to chelation therapy. Magnetic resonance imaging (MRI) has been proved effective in detecting and quantifying iron in the heart and liver.

Objective To assess the value of MRI T2* procedure in assessment of hepatic (LIC) serum ferritin level and MRI T2* of liver and myocardium in patients with BTM.

Methods A cross -sectional study was conducted at Al-Imamein Al-Kadhimein Medical City in Baghdad, from September 2016 to September 2017, 45 patients with BTM were collected from the Thalassemia center in Ibn AL-Baldy Hospital. Hepatic and myocardial T2* MRI results were taken from the file records of the patients from the same center. The results of hepatitis $B$ and $C$ and serum ferritin were taken from the file records.

Results The total number of patients was 60, 15 patients were excluded because of heart failure and hepatitis $B$ and $C$, the mean age was (19.95 \pm 6.85 years), male: female ratio equal to $1: 1$. Fifty-one patients (85\%) were on Deferasirox and 19 patients $(31.76 \%)$ were splenectomized. Serum ferritin ranged between (1009-10600 ng/l). Liver T2* MRI ranged from (0.5-17 ms) with a mean of $(3.66 \pm 3.13 \mathrm{~ms})$. Mild-moderate severity found in the majority, 18 cases $(40 \%), 13$ cases $(28.9 \%)$ respectively, with strong inverse correlation between liver MRI and serum ferritin level. Myocardial T2* MRI ranged from (1.88-33.2 ms) with a mean of (13.16 $\pm 7.81 \mathrm{~ms})$. Moderate-severe degree of severity in the majority, 12 cases $(26.67 \%), 19$ cases $(42.22 \%)$ respectively, with significant inverse correlation between serum ferritin and myocardial MRI. There is no positive correlation between myocardial T2* MRI nor liver T2* MRI with mean age of the patients.

Conclusion The study shows that MRI is an accurate and non-invasive method to assess iron overload in liver and heart in $\beta$-thalassemia patients with regular transfusion.

Keywords $\quad \beta$-Thalassemia major, blood transfusion, serum ferritin, cardiac MRI, hepatic MRI

Citation Yaseen AK, Abbas SS, Abdulhadi AMA. Correlation between cardiac and hepatic T2* MRI and serum ferritin level in patients with transfusion dependent $\beta$-thalassemia major. Iraqi JMS. 2019; 17(1): 66-73. doi: 10.22578/IJMS.17.1.10
\end{abstract}

List of abbreviations: $B$ TM $=\beta$ - thalassemia major, $F O V=$ Field of view, $\mathrm{Hb}=$ Hemoglobin, $\mathrm{LIC}=$ hepatic iron concentration, $\mathrm{MIC}=$ Myocardial iron concentration, MRI = Magnetic resonance imaging, R2 $=$ Relaxation rate, $\mathrm{SIR}=$ Signal intensity ratio, $\mathrm{T} 2 *$ : Transverse relaxation rate two star

\section{Introduction}

-Thalassemia occurs in higher frequency in the Mediterranean area and the Middle East ${ }^{(1)}$. Infants with severe beta 
thalassemia major (BTM) are well at birth. Symptoms emerge during the second six months of life when fetal hemoglobin $(\mathrm{HbF})$ is replaced by adult hemoglobin ( $\mathrm{HbA}){ }^{(2)}$. In transfused patients with BTM, cardiac hemosiderosis is the most feared complication. Without early institution of iron chelation therapy, they develop a sterile pericarditis, ventricular arrhythmias, end-stage restrictive cardiomyopathy, heart failure, and death ${ }^{(3)}$.

Hepatomegaly is prominent early in the disease, even in the absence of transfusion, the accelerated rate of erythropoiesis enhances dietary iron absorption from the gut, resulting in a chronic state of iron overload, with liver fibrosis and, potentially, end-stage liver disease (4). Serum ferritin levels in those with BTM may be quite elevated, reflecting the presence of iron overload ${ }^{(5)}$.

Magnetic resonance imaging (MRI) methods for assessing tissue iron can be separated into two groups: signal intensity ratio (SIR) methods and relaxometry methods (R2) ${ }^{(6)}$. R2 of the liver demonstrates a significant positive correlation with serum ferritin and hepatic iron concentration (LIC) determined from liver biopsy material ${ }^{(7)}$.

Myocardial iron has been evaluated by MRI using SIR and relaxometry techniques. MRI studies have demonstrated discordant results regarding the relationship of myocardial iron with hepatic iron and serum ferritin (8).

MRI studies have shown that cardiac siderosis increases with age, but that siderosis progresses more slowly in the heart than in the liver ${ }^{(9)}$.

This study aimed to assess the value of MRI T2* procedure in assessment of hepatic (LIC) and myocardial iron concentration (MIC). Also, to assess the correlation between serum ferritin and T2* MRI of liver as well as the correlation of serum ferritin and T2* MRI of myocardium.

\section{Methods}

A cross-sectional study was conducted at AL- AlImamein Al-Kadhimein Medical City in Baghdad, from September 2016 to September 2017. Cases were taken from Ibn Al-Balady hospital - thalassemia center including all the patients who had been diagnosed as BTM with specific inclusion criteria at the previous time period, they were older than 5 years and transfusion dependent. The total number of cases was 60 patients. Each patient was inquired for his name, sex, age, chelating agents used and whether splenectomy was done. All the included patients had to fulfill the following criteria: BTM patient of more than 5 years old, and were receiving regular transfusion of packed red blood cells $10-15 \mathrm{ml}$ per $\mathrm{Kg}$ body weight at 2-4 weeks interval (in order to maintain their hemoglobin level above 10 $m g \backslash d l)$, without any incidence of hepatitis B or C or any congenital or acquired liver diseases and who didn't develop heart failure. Screening for hepatitis $B$ and $C$ were taken from the file records of the patients.

For each patient included in the study, a T2* MRI study (for assessment of iron overload in the liver and the heart) had to be documented, the results were taken from the file records of the patients, for the patients who didn't have the required MRI study, researcher had arranged an MRI appointment for them in the Radiology Department of Al-Imamein AlKadhimein Medical City, furthermore, serum ferritin study was conducted monthly for each of them. Measurements were carried out by an enzyme-linked immune florescent assay, (BiomeriEux closed system Minividas apparatus, French). The results were taken from the file records of the patients. Each case was sent for ECHO study to exclude any patients with evidence of heart failure.

The exclusion criteria were any patients younger than 5 years, and patients with associated hepatitis B or C, to avoid any other parenchymal changes that can alter our results. Ethical committee approval had been taken to perform MRI study for the included patients.

\section{MRI of liver}

MRI studies performed for the elected BTM patients with a $1.5 \mathrm{~T}$ Avanto (magneto) system (Siemens medical System). Three transverse images were recorded using a body matrix coil, 
visualizing the right liver lobe, and posterior vertebral muscles in the same slice.

\section{MRI of heart}

MRI studies performed for the elected BTM patients with a $1.5 \mathrm{~T}$ Avanto (magneto) system (Siemens medical System). Three midventricular short axis slices were imaged with a slice thickness of $10 \mathrm{~mm}$ and a slice gap of 0.6 $\mathrm{mm}$. using body matrix coil, gradient echo sequence $\left(\mathrm{T} 2^{*}\right)$ with 8 echo times (3.6-16 ms) was used to obtain the images. The field of view was $400 \mathrm{~mm}$ with the Field of view (FOV) was $75 \%$, with prospective ECG triggering. The images were acquired at the mid-diastolic phase whereas the heart motion was minimal.

Normal values of MRI T2* of Liver and Heart to measure iron overload (Table 1 ).

Table 1. Normal values of MRI T2* of Liver and Heart to measure iron overload (8)

\begin{tabular}{ccccc}
\hline & Normal/ms & Mild/ms & Moderate/ms & Severe/ms \\
\hline T2* Heart & $>20$ & $14-20$ & $10-14$ & $<10$ \\
T2* Liver & $>6.3$ & $2.8-6-3$ & $1.4-2.7$ & $<1.4$ \\
\hline
\end{tabular}

\section{Statistical analysis}

Data were analyzed by IBM SPSS statistics version 24; spearman test had been used to correlate $\mathrm{S}$. ferritin level, liver $\mathrm{T} 2 *$ in $\mathrm{ms}$, cardiac $\mathrm{T} 2 *$ value in $\mathrm{ms}$. Chi square test and paired sample T-Test with Bootstrap to assess P. value, a value of $>0.05$ is considered significant. Descriptive variables were presented as numbers and percentage, continuous variables were presented as mean and standard deviation.

\section{Results}

Sixty patients were included in this cross sectional study, their mean age was (19.95 \pm $6.85)$ years distributed in numbers (Table 2 ), 31 cases (52\%) were females and 29 (52\%) were males, male:female ratio $1: 1$. All are BTM, on regular blood transfusion and on regular iron chelating agent for more than 18 months, 15 of the patients were excluded from the study because 11 was hepatitis $C$ positive and 4 had with heart failure. MRI was done for 45 patients only.

Table 2. Age distribution of the patients

\begin{tabular}{ccc}
\hline Age (year) & No. & $\%$ \\
\hline$<10$ & 2 & 3 \\
$10-14$ & 11 & 18 \\
$15-19$ & 21 & 35 \\
$20-23$ & 13 & 22 \\
$>24$ & 13 & 22 \\
\hline Total & 60 & 100 \\
\hline
\end{tabular}

Fourteen patients had undergone splenectomy (23.33\%) and fifty-one patients (85\%) were on Deferasirox (Exjade), only five of the sixty patients (8.3\%) were on Deferoxamine (Desferal) and four patients (6.7\%) stopped the treatment as shown in (Table 3). 
Table 3. Type of chelating agents

\begin{tabular}{ccc}
\hline Type of chelating therapy & No. & $\%$ \\
\hline Deferasirox & 51 & 85 \\
Deferoxamine & 5 & 8.3 \\
Non-compliant & 4 & 6.7 \\
\hline
\end{tabular}

The serum ferritin range between (1009-10600) $\mathrm{ng} / \mathrm{l}$ with mean (3737.16 \pm 2759.56$) \mathrm{ng} / \mathrm{l}$. Correlation of liver T2* MRI with serum ferritin and mean age shown in (Table 4), the majority had mild to moderate degree of severity.

Table 4. Correlation of liver T2* MRI with serum ferritin and mean age

\begin{tabular}{ccccc}
\hline $\begin{array}{c}\text { Liver T2* } \\
\text { MRI }\end{array}$ & No. (\%) & Age (year) & MRI liver (ms) & S. Ferritin (ng/l) \\
\hline Normal & $5(11.1)$ & $24.8 \pm 4.88$ & $10.48 \pm 3.8$ & $2351.0 \pm 1945.63$ \\
Mild & $18(40.0)$ & $22.94 \pm 7.13$ & $4.41 \pm 1.11$ & $2238.28 \pm 1994.29$ \\
Moderate & $13(28.9)$ & $17.0 \pm 6.1$ & $1.86 \pm 0.36$ & $3898.77 \pm 1783.64$ \\
Severe & $9(20.0)$ & $16.89 \pm 3.72$ & $0.97 \pm 0.34$ & $7271.56 \pm 2532.85$ \\
\hline Total & $45(100)$ & $20.22 \pm 6.72$ & $3.66 \pm 3.13$ & $3737.16 \pm 2759.56$ \\
\hline r. value & & & & -0.528 \\
P. value & & & & $<0.001$ \\
\hline
\end{tabular}

There was strong inverse correlation between liver MRI and serum ferritin level $(r=-0.528)$ with significant $P$ value of $<0.001$. as it is shown in (Figure 1).

Correlation of myocardial T2* MRI with serum ferritin and mean age is shown in (Table 5), the majority had moderate to severe degree of severity.
There was strong inverse correlation between myocardial MRI and S. ferritin $(r=-0.468)$ with significant $P$ value of 0.001 (Figure-2).

The mean myocardial T2* MRI was (13.16 \pm 7.81 ), While the mean liver T2* MRI was (10.48 \pm 3.8$) \mathrm{ms}$, with a discordant result regarding the relationship between myocardial iron content and hepatic iron content (Figure 3). 


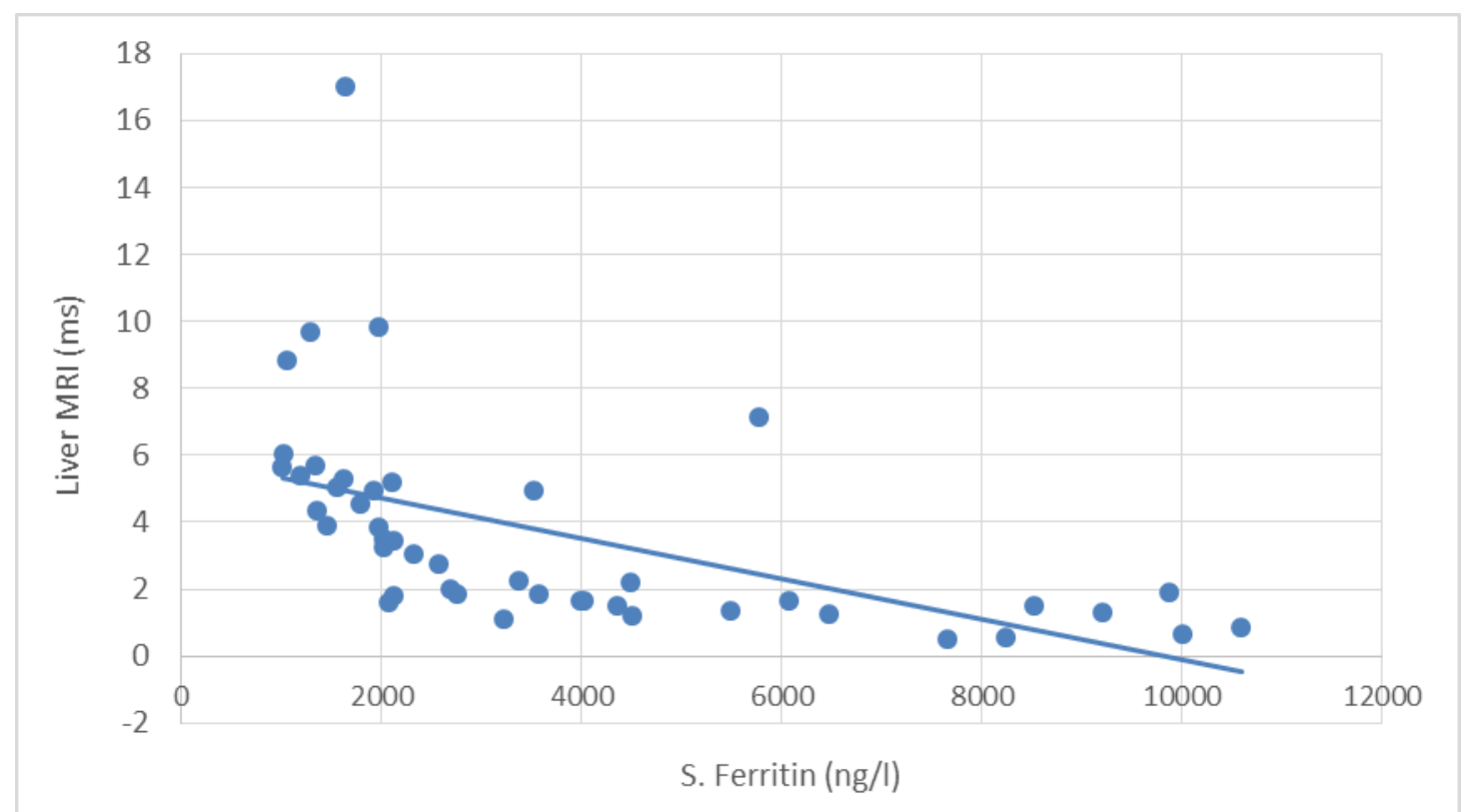

Figure 1. Relationship between liver $\mathrm{T}^{*} * \mathrm{MRI}$ and serum ferritin

Table 5. Correlation of myocardial T2* MRI with serum ferritin and mean age

\begin{tabular}{ccccc}
\hline $\begin{array}{c}\text { Myocardial } \\
\text { T2* MRI }\end{array}$ & No. (\%) & Age (year) & MRI liver (ms) & S. Ferritin (ng/l) \\
\hline Normal & $10(22.2)$ & $21.7 \pm 6.38$ & $25.05 \pm 4.36$ & $2882.3 \pm 2843.73$ \\
Mild & $4(8.89)$ & $22.5 \pm 6.95$ & $16.62 \pm 1.05$ & $2149.0 \pm 428.15$ \\
Moderate & $12(26.68)$ & $19.83 \pm 8.19$ & $12.95 \pm 1.07$ & $2380.75 \pm 1334.9$ \\
Severe & $19(42.23)$ & $19.21 \pm 6.13$ & $6.31 \pm 2.18$ & $5378.11 \pm 2901.13$ \\
\hline Total & $45(100)$ & $20.22 \pm 6.72$ & $13.16 \pm 7.81$ & $3737.16 \pm 2759.5$ \\
\hline r. value & & & & -0.468 \\
P. value & & & & 0.001 \\
\hline
\end{tabular}




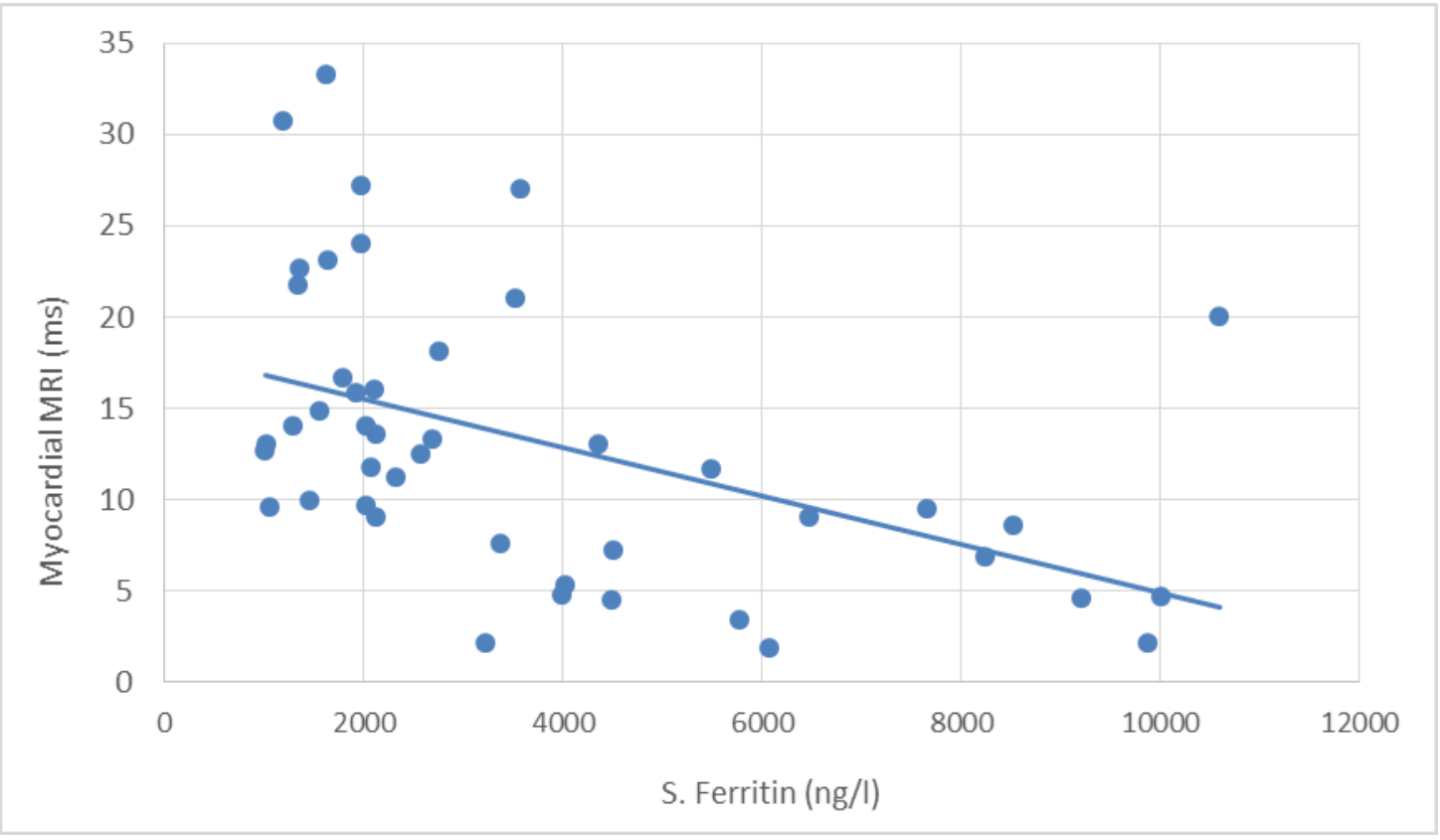

Figure 2. Relationship between myocardial T2* MRI and serum ferritin

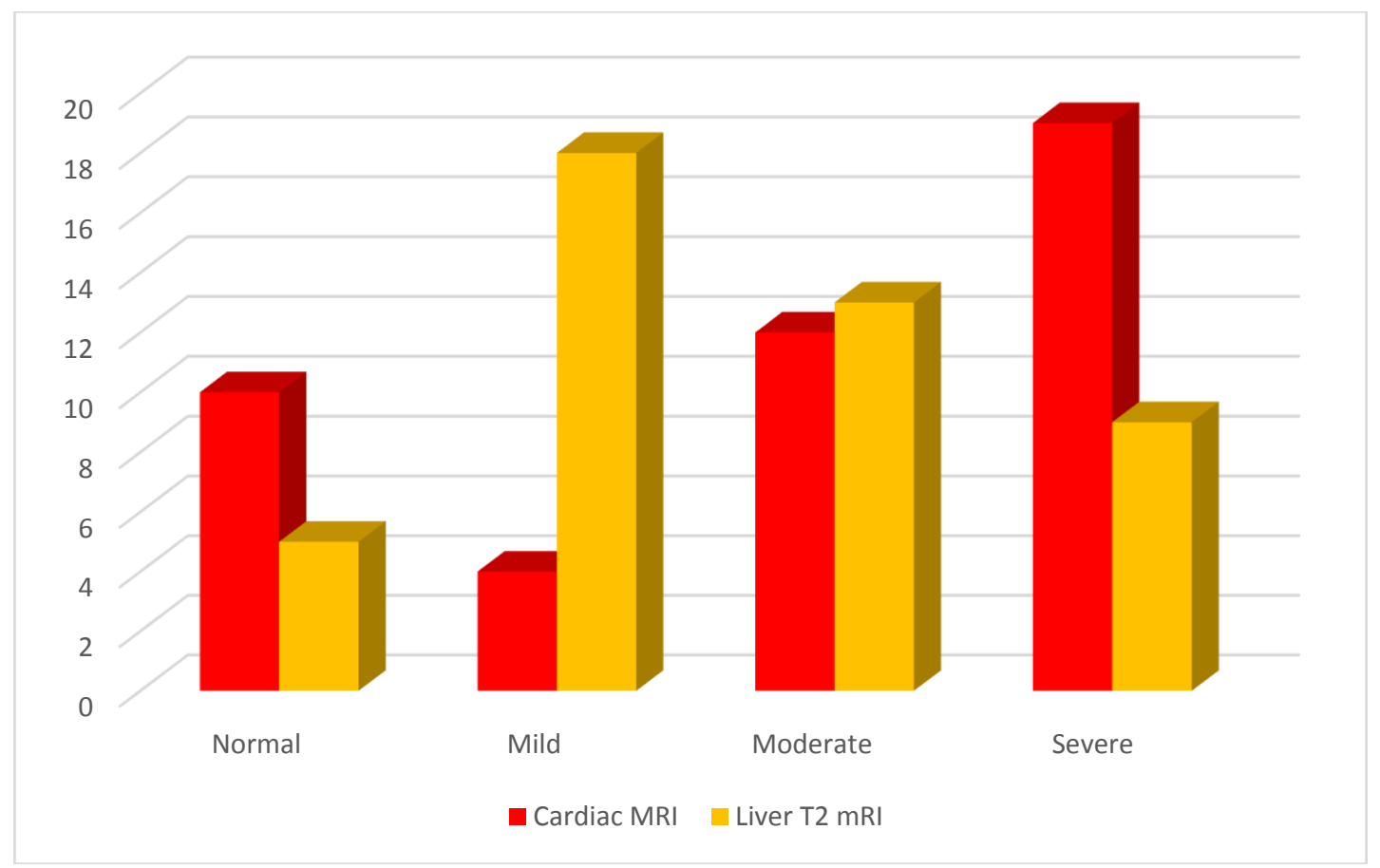

Figure 3. Difference between liver and myocardial $T 2 * M R I$ in the study group 


\section{Discussion}

Regarding the distribution of severity of iron overload in hepatic and cardiac T2 MRI finding, 14 cases (31\%) were of normal to mild severity in cardiac MRI where 31 cases (69\%) were of moderate to severe degree in cardiac MRI, on other hand; 23 (51\%) cases were of mild to moderate severity and the rest 22 (49\%) cases were of moderate to severe degree, which is the same outcome of Noetzli et al. study in Tehran, which shows that there is no correlation between cardiac and hepatic T2 MRI in relation to the severity of the disease ${ }^{(11)}$.

Correlating hepatic MRI finding with the severity of the disease, a relatively small number of cases (9 cases; 20\%) were with severe degree of hepatic MRI finding, which may be due to the fact that the chelating agents acting more efficiently on the liver with easy excretion of iron overload from the liver than that of the heart.

In the present study, analyzing of correlation between hepatic T2 MRI finding and the serum ferritin, the study shows that there is significant correlation between them with a $P$ value of $<0.001$, which is the same finding in Majd et al. study in Iran (10), Eghbali et al. study (12), and Zamani et al. study in Iran ${ }^{(13)}$. Such results can be accepted when understanding that $70 \%$ of iron stores in the body are deposited in the liver. Regarding the correlation between cardiac T2 MRI finding and serum ferritin level, the study shows that there is strong correlation between them with a $P$ value equal to 0.001 , which is also significant in Majd et al. study in Iran ${ }^{(10)}$ and Kirk et al. study in UK (14). Such finding can be explained due to iron overload toxicity in the heart that occurs in disease process; which is associated with many cardiac complications such as heart failure and arrhythmias which are the main causes of death in patients with BTM. This finding is in contrast to Azarkeivan et al. study in Iran, which shows there is no significant correlation between serum ferritin level and cardiac T2 MRI, which is explained by documentation of paradoxical low serum ferritin level in certain patients with heavy iron overload ${ }^{(15)}$.

Differences in iron kinetics and variations in chelation schemes may be responsible for the lack of correlation of the MRI-determined myocardial iron with that of the liver. More active elimination of iron from the hepatocytes than from the myocytes may play a role in the absence of correlation in patients receiving chelation therapy ${ }^{(8)}$.

Splenectomy was done for 15 out of 45 cases (33\%) cases, while in Vichinsky et al. study, $45 \%$ of 407 cases had been splenectomized ${ }^{(16)}$, while in Fahmy et al. study, 12 cases (17\%) of the seventy cases had been splenectomized ${ }^{(17)}$.

This study concluded that there is strong correlation between serum ferritin and liver and heart MRI findings, so we can depend on serum ferritin in centers which lack MRI technique to assess the iron overload in heart and liver.

MRI is accurate and non-invasive method to assess iron over load in the vital organ (heart and liver), in $\beta$ - thalassemia patients who are on regular transfusion.

This study recommends:

1. A larger study to find the correlation between serum ferritin and iron overload in heart, liver, pituitary gland and pancreas.

2. Using MRI as a yearly standard follow up in $\beta$ thalassemia patients with regular transfusion who are at high risk with iron overload so we can early detect the complications of the organs that suffer from iron overload.

\section{Acknowledgments}

To all children with $\beta$-thalassemia who fight for their survival.

\section{Author contribution}

All authors contributed to this manuscript. They coordinated study recruitment, implementation and progress of this study and helped with data interpretation and manuscript organization and editing.

\section{Conflict of interest}

The authors have no conflict of interest.

\section{Funding}

Self- funding.

\section{References}

1. Vichinsky EP. Changing patterns of thalassemia worldwide. Ann N Y Acad Sci. 2005; 1054: 18-24.

2. Bird EM, Parameswaran $U$, William $T$, et al. Transfusion-transmitted severe Plasmodium malaria 
in a splenectomized patient with beta-thalassaemia major in Sabah, Malaysia: a case report. Malar J. 2016; 15(1): 357. doi: 10.1186/s12936-016-1398-z.

3. Aessopos A, Berdoukas V. Cardiac function and iron chelation in thalassemia major and intermedia: a review of the underlying pathophysiology and approach to chelation management. Mediterr J Hematol Infect Dis. 2009; 1(1): e2009002. doi: 10.4084/MJHID.2009.002.

4. Tanno T, Bhanu N V, Oneal PA, et al. High levels of GDF15(Growth differentiation factor) in thalassemia suppress expression of the iron regulatory protein hepcidin. Nat Med. 2007; 13(9): 1096-101. doi: $10.1038 / \mathrm{nm} 1629$.

5. Modell B, Darlison M. Global, epidemiology of haemoglobin disorders and derived service indicators. Bull World Health Organ. 2008; 86(6): 480-7.

6. Argyropoulou MI, Astrakas L. MRI evaluation of tissue iron burden in patients with beta-thalassaemia major. Pediatr Radiol. 2007; 37(12): 1191-200. doi: 10.1007/s00247-007-0567-1

7. Alexopoulou E, Stripeli F, Baras $P$, et al. $R 2$ relaxometry with MRI for the quantification of tissue iron overload in $\beta$-thalassemic patients. J Magn Reson Imaging. 2006; 23(2): 163-70. doi: 10.1002/jmri.20489

8. Pennell DJ. T2* magnetic resonance and myocardial iron in thalassemia. Ann N Y Acad Sci. 2005; 1054: 3738. doi: 10.1196/annals.1345.045.

9. Perifanis V, Christoforidis A, Vlachaki E, et al. Comparison of effects of different long-term ironchelation regimens on myocardial and hepatic iron concentrations assessed with $\mathrm{T} 2 *$ magnetic resonance imaging in patients with $\beta$-thalassemia major. Int $\mathrm{J}$ Hematol. 2007; 86(5): 385-9. doi: 10.1532/IJH97.E0734.

10. Majd Z, Haghpanah S, Ajami GH, et al. Serum ferritin levels correlation with heart and liver MRI and LIC in patients with transfusion-dependent thalassemia. Iran Red Crescent Med J. 2015; 17(4): e24959. doi: 10.5812/ircmj.17(4)2015.24959.
11. Noetzli LJ, Carson SM, Nord AS et al. Longitudinal analysis of heart and liver iron in thalassemia major. Blood. 2008; 112(7): 2973-8. doi: 10.1182/blood2008-04-148767.

12. Eghbali A, Taherahmadi $H$, Shahbazi $M$ et al. Association between serum ferritin level, cardiac and hepatic T2-star MRI in patients with $\beta$-thalassemia major. Iran J Ped Hematol Oncol. 2014; 4(1): 17-21.

13. Zamani F, Razmjou S, Akhlaghpoor S, et al. T2* magnetic resonance imaging of the liver in thalassemic patients in Iran. World J Gastroenterol. 2011; 17(4): 522-5. doi: 10.3748/wjg.v17.i4.522.

14. Kirk $P$, Roughton $M$, Porter JB, et al. Cardiac T2* magnetic resonance for prediction of cardiac complications in thalassemia major. Circulation. 2009; 120(20): 1961-8. doi: 10.1161/CIRCULATIONAHA.109.874487.

15. Azarkeivan A, Hashemieh $M$, Akhlaghpoor $S$, et al. Relation between serum ferritin and liver and heart MRI T2* in beta thalassemia major patients. East Mediterr Health J. 2013; 19(8): 727-32.

16. Vichinsky E, Neumayr L, Trimble $S$, et al. Transfusion complications in thalassemia patients: a report from the Centers for Disease Control and Prevention (CME). Transfusion. 2014; 54(4): 972-81; quiz 971. doi: 10.1111/trf.12348.

17. Fahmy HS, Khatir NH, El-Shahat HM, et al. Reassessing the value of MRI T2* in evaluation of hepatic and myocardial iron concentration: an institutional study. Egypt J Radiol Nuclear Med. 2015; 46(4), 1085-90. doi: https://doi.org/10.1016/j.ejrnm.2015.06.008.

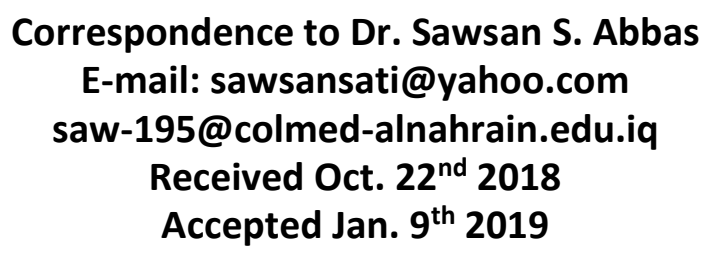

